survey contained questions about demographics, choice of position during LPs, reasons for that choice, use and frequency of analgesia, LP training background and demand for training. Questions about positions and pain relief were divided into different age groups.

Results A total of 84 questionnaires were completed, which demonstrated that the most common position being used in children under the age of 1 was the lateral recumbent position with neck flexion $(83 \%$ in newborns to 3 months and $59 \%$ in 3 months to 1 year). $61 \%$ of participants said this position was used to increase the interspinous distance, whilst $27 \%$ said is was used to best hold the child still. Sucrose was the most commonly used for of pain relief in children under one, however $39 \%$ of participants never, rarely or only sometimes used pain relief in this age group. $79 \%$ of participants would appreciate more training in this area.

Conclusion We demonstrated that a painful, uncomfortable and potentially dangerous position to hold children during LPs was the first choice in the majority of cases. We also demonstrated that the use of analgesia in general was either absent or poor. Further education of healthcare staff involved in this procedure is needed.

\section{G135(P) DEVELOPMENT OF CHILDREN AND YOUNG PEOPLE'S ASSESSMENT SERVICE STANDARDS AND A SUPPORTIVE PEER REVIEW PROCESS ACROSS A REGION}

doi:10.1136/archdischild-2013-304107.147

${ }^{1,2,3} \mathrm{M}$ Clements, ${ }^{1,3} \mathrm{~K}$ Evans, ${ }^{1,4} \mathrm{C}$ Upton. 'Strategic Network of Child Health and WellBeing, NHS East of UK, Cambridge, UK; ${ }^{2}$ Department of Paediatrics, West Suffolk Hospital NHS Foundation Trust, Bury St Edmunds, UK; ${ }^{3}$ Children and Young People programme, NHS Institute for Innovation and Improvement, Coventry, UK; ${ }^{4}$ Department of Paediatrics, Norfolk and Norwich University Hospital NHS Foundation Trust, Norwich, UK

Aims To develop a set of standards and a peer review process for Children and Young People's (CYP's) ambulatory care.

Methods Development of the standards involved reviewing national, regional and local policies/best practise. Questionnaires were circulated and focus groups facilitated with multidisciplinary staff involved in CYP ambulatory care across a region. Professionals challenged and tested the standards as they evolved. This included CYP and family feedback and enagement.

All 17 acute trusts involved in CYP acute care in the region performed a self-assessment using the assessment service standard tool and participated in an external peer review visit.

The visit started with informal discussions with staff and service users. The team walked the patient pathway. A whole-system professionals meeting, involving senior Managers/Executives, Clinicians/Senior Nursing team, Play Specialists and Commissioners concluded the visit. The local team presented their self-assessment and the visiting team feedback their findings leading to a discussion/action planning for next steps.

The process was evaluated.

Each organisation showcased the good practise identified at a celebration event.

Re-visits to all trusts are arranged to review progress and assess against the national standards for CYP in the emergency department. Results A supportive process for peer review for children and Young People's assessment services and emergency and urgent care, including comprehensive standards have been developed and evaluated.

A poster summarises feedback from Children, young people and families. Figure 1

Current practise for ambulatory care across a region has been reviewed and key findings from the visits including the variety of models of care, good practise and recommendations summarised and shared.

The visits were evaluated as supportive and useful in raising the profile of CYP within Trusts. The standards gave a focus for organisations to review their services and plan improvements.
There was evidence the standards had driven improvements and good practise identified in every unit.

Conclusion There is already evidence showing standards and peer review improves quality of care.

We have developed a processes and tools that can be used nationally for CYP's emergency and urgent care

These visits were not mandatory but $100 \%$ of Trusts participated in the self-assessment and peer review and booked re-visits.

\section{G136(P) IMMUNISATION OF HIV POSITIVE CHILDREN}

doi:10.1136/archdischild-2013-304107.148

${ }^{1}$ A Bailey, ${ }^{2}$ S Bandi. 'Department of Paediatrics, LNR Deanery, Leicester, UK; '2Leicester Children's Hospital, Leicester Royal Infirmary, Leicester, UK

Aims To determine if the immunisation schedules of thirty one children attending a tertiary paediatric HIV clinic in 2012 adhered to the Children's HIV association guidelines on immunisation in HIV positive children.

Methods We looked at the immunisation records of the thirty one children attending the tertiary paediatric HIV clinic. A standard proforma was used for data collection from the health care records, clinic letters and 'Red Book'. We also contacted the General Practice (GP) surgeries for those with inadequate information in the health records. Results Children were aged between four and sixteen years with a fairly even distribution between sexes. 25/31 children were born abroad. Children were classed as fully immunised if they were vaccinated according to UKguidelines. Overall $48 \%$ received complete primary and $38 \%$ received complete booster immunisations. $83 \%$ of immunisations were complete in UK born children compared with $28 \%$ in non-UK born children. We were unable to obtain immunisation information in $25 \%$ of children who were born abroad, there was no information regarding immunisation status in either health records or in GP surgery records.

Figure 1 details the immunisation details for all our children. In addition, Human Papilloma Virus (HPV) vaccine was given in $56 \%$ of eligible patients. $52 \%$ of patients received a BCG vaccine and all of them were born abroad. $48 \%$ received an annual influenza vaccine and $68 \%$ of children had received the Hepatitis $B$ vaccine.

Conclusions There is poor vaccination of children with HIV, especially those born abroad and there is an urgent need for strategies to be implemented in order to achieve better rates of immunisation. Recommendations include interface between hospitals and GP practises with improved access to immunisation records, reminder letters to GPs and families and possible opportunistic immunisation in hospitals.

\section{G137(P) HOW TO SAVE A SMILE!}

doi:10.1136/archdischild-2013-304107.149

${ }^{1} \mathrm{KG}$ Greenwood, 'DT Tuthill, ' $\mathrm{CP}$ Patel, ${ }^{2} \mathrm{EM}$ Hingston. 'General Paediatrics, Cardiff and Vale UHB, Cardiff, UK; ${ }^{2}$ Paediatric Dentistry, Cardiff and Vale UHB, Cardiff, UK

Introduction Dental caries is entirely preventable, yet remains the most common chronic disease of childhood- affecting $50 \%$ of children under five in Wales. It is caused by acid produced by the bacterial metabolism of dietary carbohydrates, particularly refined sugars. Frequent squeal include: pain, infection, poor aesthetics and permanent teeth damage. Basic standards of advice to prevent dental caries exist. ${ }^{1}$ Ensuring medical staff have the knowledge to advocate appropriate oral care, and know when to refer to dental colleagues could reduce the burden of dental disease.

Aims To audit knowledge of staff regarding dental health care in children against basic dental guidelines ${ }^{1}$.

Methods Structured questionnaires were designed by paediatricians and dentists and administered to healthcare staff. These were based upon: dietary advice including limitation of fruit juices, 